\title{
Rare Occurrence of Single Rooted Mandibular Left First and Third Permanent Mandibular Molar: A Case Report
}

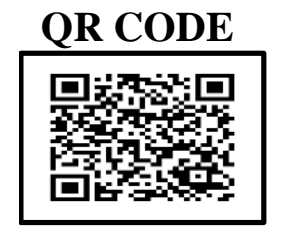

\author{
NEHA BANSAL ${ }^{* 1}$, HIMANSHU MEHTA', TUSHAR RATHOR', NALINI TRIVEDI'
}

For any practicing clinician, successful endodontic therapy is highly dependent upon complete knowledge of the anatomy and the variations present in the human dentition. There are variances in anatomical configurations in teeth seen across the globe and might needs specialized treatment techniques. The tendency of Asians to show a C-shaped canal morphology has been documented in the literature, however, in contrast to those findings, we report a unique and a rare case of a left mandibular first and third molar having a single root with single root canal morphology in a middle aged Indian female.

KEYWORDS: First Molar, Third Molar, Single canal, Single Root

\section{INTRODUCTION}

A dental clinician aims to alleviate the pain of his patients sitting in his dental chair. While certain endodontic procedures are simple to perform due to a known root and canal anatomy, variations in them can pose a serious challenge to these clinicians. Literature relating to reporting of complicated root canal systems rather than simplified canals have been described until the year 1925, and has changed the face of modern endodontics ever since. ${ }^{1}$

The permanent mandibular first molar usually exhibits two roots (one mesial and other distal) with three root canals and variations in the number and configuration of both roots as well as root canals have been extensively documented by various clinicians. ${ }^{2}$ The roots can fuse to form a single conical root with varying internal anatomy and often have $\mathrm{C}$-shaped canal configuration and is mostly seen among Chinese, Korean and Indian populations. ${ }^{3,4}$

Therefore, it becomes clear that to treat teeth requiring endodontic treatment, a correct diagnosis (involving the use of X-rays) coupled with appropriate cleaning and shaping of the root canal system including any accessory canal leads to a successful endodontic treatment outcome. ${ }^{5}$ Slowey (1974), reports that a major cause of endodontic treatment failures has been attributed to a lack of understanding as well as underestimation of the root canal morphology by clinicians across the globe. ${ }^{6}$

When it comes to the documentation of aberrant root forms, the presence of a single, tapering root form can be found in any molar, most common being second and third permanent molars which shows a female predilection, for which the cause is still being investigated by researchers. ${ }^{7}$

We present a rare case report, showing the bilateral presence Vertucci's type I (1-1) root canal, having a single root canal with one orifice and one apical foramen in the mandibular left first and third permanent first molar.8 It is of great clinical importance to note that our case had a single root and a single root canal morphology, which is quite unique and rare.

\section{CASE REPORT}

A 46 year old female patient reported to the OPD of GTB hospital, New Delhi complaining of pain in her lower left back tooth region since 2 days which was extending upto her forehead and was constant in nature. The pain aggravated upon eating anything hot or cold and relief was attained only with medication (analgesic). The medical history of the patient was non-contributory. Upon clinical examination, the left permanent mandibular first molar (tooth number 36 ) showed no frank carious lesion but tooth number 36 was tender on percussion. An IOPA was taken to check for periapical pathology, which was found to be absent (Figure 1). The IOPA further revealed occlusal caries (radiolucency) from the mesial and distal aspect of tooth number 36 and affecting tooth number 35 from the proximal aspect near the dentino-enamel junction. The apical portion of tooth number 36 showed a conical configuration.

Further analysis revealed that tooth number 38 also 


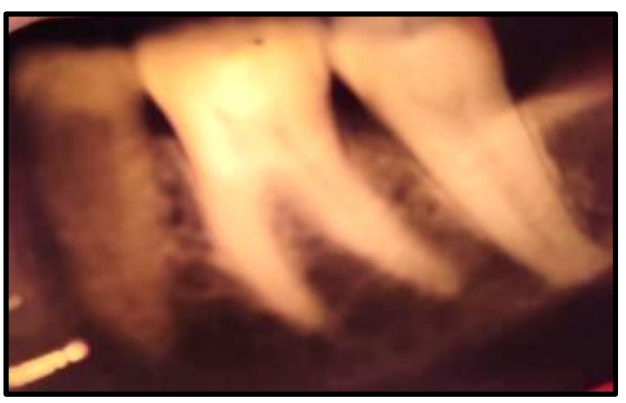

Figure 1. IOPA taken of the affected tooth number 36 revealed a single root and canal in tooth number 38 too.

had a single root profile with a single Vertucci's type I (1-1) root canal configuration and was non-tender, non-carious and revealed a non-conical root configuration with a clinically corelateable pocket of 4 $\mathrm{mm}$. The patient was advised to undergo both periodontal (root planning w.r.t 38) and endodontic treatment for tooth number 36 , but she declined to provide any consent for treatment or any blood investigations. The patient was recalled for counselling regarding the condition of the teeth, but she did not report back to the clinic and telephonic follow up revealed that the patient had gotten the tooth extracted from a local village practitioner.

The patient's both maxillary and mandibular arches were partially edentulous in nature and had all her other molars missing and reported getting them extracted due to caries and this claim, however, could not be verified. She also could not provide any records for the extracted teeth (OPD card and any previous IOPA) to check for any bilateral presence of such a condition.

\section{DISCUSSION}

Variations in the anatomy of the configuration of mandibular molars is quite common with Asians reporting a high frequency of single rooted mandibular second molars. 9 Such variations are attributed to disturbances occurring during the canal differentiation phase. ${ }^{10}$

The present case report documents the presence of a single root and canal with a conical (First molar) and non-conical (Third molar) configuration in both mandibular left quadrant of a patient aged 6 years and these findings make the case quite rare in nature.
Munavalli A et al. ${ }^{10}$ in their case report highlighted the presence of a rare anatomy in mandibular first molar having a single root and a single canal while Thakar SS et al. ${ }^{3}$ documented bilateral single root and single canal in mandibular second molars.

Sabala et al. (1994), stated that the rarer the aberration, there are higher changes of it being bilateral in nature.11 In the present case, since all the molars were missing, we were unable to assess the finding of the current patient being bilateral or not.

In cases where only one canal is present, its location usually will be in the centre of the root. It is important to take note that a root always has a root canal, even in the most complex cases where the canal is not visible on the radiograph and is difficult to locate as well as negotiate. ${ }^{12}$ One of the key factors in the success of endodontic therapy remains instrumentation, hence, a dentist should try to be aware of all the anatomical variants and aberrant canal configurations present in human teeth. A thorough examination of the pulp chamber and ensuring complete debridement of all the canals increases the chance for long-term successful endodontic therapy. ${ }^{13}$

\section{CONCLUSION}

The present case report depicts a rare a left mandibular first and third molar having a single root with single root canal morphology in a middle aged Indian female. A keen eye on such occurrences helped us report this case. We hope that this case report shall immensely help our peers as we bring forth a clinical anomaly for them to discuss and relate to in the near future.

\section{REFERENCES}

1. Hess W, Zurcher E. The Anatomy of the Root Canals of the Teeth of the Permanent Dentition and the Anatomy of the Root Canals of the Deciduous Dentition and the First Permanent Molars. London: Basle, Sons and Danielson; 1925.

2. Nagaveni N B, Manoharan M, Yadav S, Poornima P. Single rooted, single canalled mandibular first molar in association with multiple anomalies: Report of a rarest case with literature review. Niger J Exp Clin Biosci 2015;3:59-63.

3. Thakar SS, Motghare V, Prabhakar I, Shivlingesh KK, Gupta B, Gupta N. Bilateral Presence of a Single Root in Mandibular Second Molars having a Single NonConical Canal Configuration: A Rare Case Report. Int J 
Adv Health Sci 2014; 1(3): 31-4.

4. Seo MS, Park DS. C-shaped root canals of mandibular second molars in a Korean population: clinical observation and in vitro analysis. Int Endod J. 2004 Feb; 37(2): 139-44.

5. Fava LR, Weinfeld I, Fabri FP, Pais CR. Four second molars with single roots and single canals in the same patient. Int Endod J 2002;33(2):138-42. 6. Slowey RR. Radiographic aids in the detection of extra root canals. Oral Surg Oral Med Oral Pathol 1974;37:762-72.

7. Jeevanandan G, Subramanian E, Muthu MS. Singlerooted primary first molars. Indian J Dent Res 2012;23:104-6.

8. Vertucci FJ. Root canal anatomy of the human permanent teeth. Oral Surg Oral Med Oral Pathol.1984;58(5):589-99.
9. Manning SA. Root canal anatomy of mandibular second molars. Part II. C-shaped canals. Int Endod J 1990;23(1):40-5.

10. Munavalli A, Kambale S, Ramesh S, Ajgaonkar N. Mandibular first molar with single root and single root canal. J Conserv Dent. 2015; 18(4):346-8. doi: 10.4103/0972-0707.159757

11. Sabala CL, Benenati FW, Neas BR. Bilateral root or root canal aberrations in a dental school patient population. J Endod 1994;20(1):38-42.

12. Vertucci FJ. Root canal morphology and its relationship to endodontic procedures. Endod Top 2005; 10(1): 3-29.

13. Baugh D, Wallace J. Middle mesial canal of the mandibular first molar: a case report and literature review. J Endod 2004; 30(3): 185-6. 